\title{
Quantification of cancer cell migration with an integrated
}

\section{experimental-computational pipeline [version 1; peer review:}

\section{2 approved with reservations]}

\author{
Edwin F Juarez (Did) 1 , Carolina Garri1 , Ahmadreza Ghaffarizadeh1, \\ Paul Macklin (D1,4, Kian Kani1 \\ ${ }^{1}$ Lawrence J. Ellison Institute for Transformative Medicine, Keck School of Medicine, University of Southern California, Los Angeles, \\ California, 90007, USA \\ 2Department of Medicine, University of California San Diego, La Jolla, California, 92093, USA \\ ${ }^{3}$ Ming Hsieh Department of Electrical Engineering, University of Southern California, Los Angeles, California, 90007, USA \\ ${ }^{4}$ Intelligent Systems Engineering, Indiana University, Bloomington, Indiana, 47405, USA
}

V1 First published: 15 Aug 2018, 7:1296

https://doi.org/10.12688/f1000research.15599.1

Latest published: 15 Aug 2018, 7:1296

https://doi.org/10.12688/f1000research.15599.1

\section{Open Peer Review}

Approval Status ? ?

1

2

$\begin{array}{lll}\text { version } 1 & ?\end{array}$

15 Aug 2018

view

view

1. Mohammed El-Kebir ID, University of Illinois at Urbana-Champaign, Urbana, USA

2. Assaf Zaritsky iD, Ben-Gurion University of the Negev, Be'er Sheva, Israel

Any reports and responses or comments on the article can be found at the end of the article.

Keywords

Migration, Quantification, User-Friendly, Microscopy. 


\section{Corresponding author: Edwin FJuarez (ejuarez@ucsd.edu)}

Author roles: Juarez EF: Conceptualization, Data Curation, Formal Analysis, Investigation, Methodology, Project Administration, Resources, Software, Validation, Visualization, Writing - Original Draft Preparation, Writing - Review \& Editing; Garri C:

Conceptualization, Data Curation, Formal Analysis, Investigation, Methodology, Resources, Validation, Visualization, Writing - Review \& Editing; Ghaffarizadeh A: Conceptualization, Formal Analysis, Investigation, Methodology, Resources, Software, Validation, Writing Review \& Editing; Macklin P: Funding Acquisition, Project Administration, Resources, Supervision, Writing - Review \& Editing; Kani K: Conceptualization, Data Curation, Investigation, Project Administration, Resources, Supervision, Writing - Review \& Editing

Competing interests: No competing interests were disclosed.

Grant information: This work was supported by the USC Center for Applied Molecular Medicine, the National Institutes of Health (Physical Sciences Oncology Center [5U54CA143907] for Multi-scale Complex Systems Transdisciplinary Analysis of Response to Therapy (MCSTART), and [1R01CA180149]), the Breast Cancer Research Foundation, the USC James H. Zumberge Research and Innovation Fund, and a USC Provost's PhD fellowship.

The funders had no role in study design, data collection and analysis, decision to publish, or preparation of the manuscript.

Copyright: $\odot 2018$ Juarez EF et al. This is an open access article distributed under the terms of the Creative Commons Attribution License , which permits unrestricted use, distribution, and reproduction in any medium, provided the original work is properly cited.

How to cite this article: Juarez EF, Garri C, Ghaffarizadeh A et al. Quantification of cancer cell migration with an integrated experimental-computational pipeline [version 1; peer review: 2 approved with reservations] F1000Research 2018, 7:1296 https://doi.org/10.12688/f1000research.15599.1

First published: 15 Aug 2018, 7:1296 https://doi.org/10.12688/f1000research.15599.1 


\section{Introduction}

In order to understand and treat cancer, we need to study and ultimately control metastasis ${ }^{1}$. A key aspect of metastasis is cell migration ${ }^{1}$. Thus, assays that can reliably provide quantitative readouts of cell migration are an important component of cancer research. Here we describe an integrated computational pipeline to quantify cell migration using fluorescent microscopy.

The anterior gradient protein 2 (AGR2) has been shown to promote cell migration ${ }^{2,3}$. High expression of AGR2 is correlated with aggressive forms of various adenocarcinomas including prostate $^{3}$ and breast cancer ${ }^{4,5}$. Therefore, AGR2 is a biomarker and therapeutic target that will provide a suitable biological readout of cell migration for our pipeline to quantify. In other words, because AGR2 is known to promote cell migration, it is ideal for testing a computational platform's ability to detect changes in cell migration.

In this work, we describe an experimental and computational pipeline to quantitate cell migration. We demonstrate this pipeline by quantifying migration of MDA-MB-231 cells, a breast cancer cell line known to migrate aggressively ${ }^{6}$. We show that blocking the extracellular AGR2 (eAGR2) with a neutralizing antibody that binds specifically to AGR2 (referred to as AGR2-Ab) in cell medium prevents the migration of the MDA-MB-231 cells ${ }^{3}$. Our pipeline aids in the verification of well-established hypotheses and it can be used to test new hypotheses, thus aiding in and accelerating the drug discovery process.

\section{Methods}

Cell culture

MDA-MB-231 cell line was obtained from the American Type Culture Collection (ATCC, No. HTB-26) and tested for mycoplasma contamination before usage. Cells were maintained in DMEM media (Thermo Fisher, No. 21063045), supplemented with $10 \%$ fetal bovine serum (FBS), they were kept at $37^{\circ} \mathrm{C}$ in a humidified incubator with $5 \% \mathrm{CO}_{2}$. Cells were used within 6 months of purchase.

\section{Cell migration assay}

The Oris ${ }^{\mathrm{TM}}$ migration assay (No. CMA1.101, Platypus Technologies, Madison, WI, USA) uses a physical barrier "stopper" to create a defined circular region that is intended to prevent cell adhesion at the start of the assay. This central cell-free detection zone is in the center of each well of a 96-well plate. As the cells migrate to the cell-free zone over 24-48 hours, real-time assessment of migratory cells allows acquisition of richer data sets. Since there are no artificial membranes or inserts in the light path through which cells must pass, this assay is amenable to quantification with microscopy. We used the Oris cell migration assay from Platypus Technologies to create migration regions by inserting stoppers in each of the 96 wells on a plate. Shortly after inserting the stoppers, we seeded MDA-MB-231 cells and waited until they reached $80 \%$ confluent (approximately 24 hours). We then fed cells with either treated or untreated media. Treated media included Taxol (5 nM), mouse anti-AGR2 antibody (1:50 of $2 \mu \mathrm{g} / \mathrm{ml}$ ) (Santa Cruz Biotechnology, No. sc-101211), and mouse-IgG (Santa
Cruz Biotechnology, No. sc-2005), while the untreated media was Dimethyl sulfoxide (DMSO) (VWR, No. 97061-250). Next we removed the stoppers and allowed the cells to move into the migration region. 48 hours after removal of the stoppers, we imaged each well using a fluorescence microscope (Zeoiss Observer.Z1 microscope at $2.5 \mathrm{x}$ objective with AxioCam MRm camera and Axio Vision 4.8 software).

\section{Implementation}

This tool reads microscopy images which are placed in the same folder as the main code. First, the script first trains using the negative control (i.e., the image of a well where the stopper was not removed) to find the optimal disk which represents the migration region. Next, the script finds all the images present in the same folder as the code (by default it looks for TIF files) and applies the migration quantification metric to each of them, recording its output in a text file called output_c3. txt for easy access and plotting (a Python 3 script which creates a publication-quality plot based on this output is provided as well for any user who wishes to use it).

\section{Operation}

Typically a user only needs to make one modification the main script called code.m, make one minor modification to the img name variable (on the third line of the script), and run the script. The change to the img_name variable needs to reflect the name of the image which contains the negative control.

If the user desires change the format of the images the script uses for the quantification, the line file_list= dir('*.tif'); needs to change to reflect the desired format. The images need to be in the same directory as the script.

This tool has been tested in multiple laptops running Matlab spanning releases 2015a-2018a.

\section{Automatic selection of migration region}

We first created a mask corresponding to the area covered by cells using standard deviation filtering and applying a series of morphological operations in Matlab R2016a (MathWorks) as shown in Figure 1.

Note that these images are gray scale (green is used throughout to highlight software outputs as is shown in the right panel of Figure 1), hence every pixel's value belongs to the interval $[0,1]$ where a completely black pixel has value 0 and a completely white pixel has value 1 . Also note that a mask is a binary matrix that indicate which pixels belong to the mask (with value of 1 , these pixels are referred to as "cell pixels") and which pixels do not belong to the mask (with value 0).

We then used a genetic algorithm to determine the coordinates of the center and the radius of a circle according to Equation (1). This optimal circle determines the migration region.

$$
\left[c_{x}^{*}, c_{y}^{*}, r^{*}\right]=\underset{c_{i}, c_{j}, r}{\operatorname{argmax}} \sum_{m_{i, j} \in \boldsymbol{M}} m_{i, j}-p\left(\# \boldsymbol{M}-\sum_{m_{i, j} \in \boldsymbol{M}} m_{i, j}\right),
$$



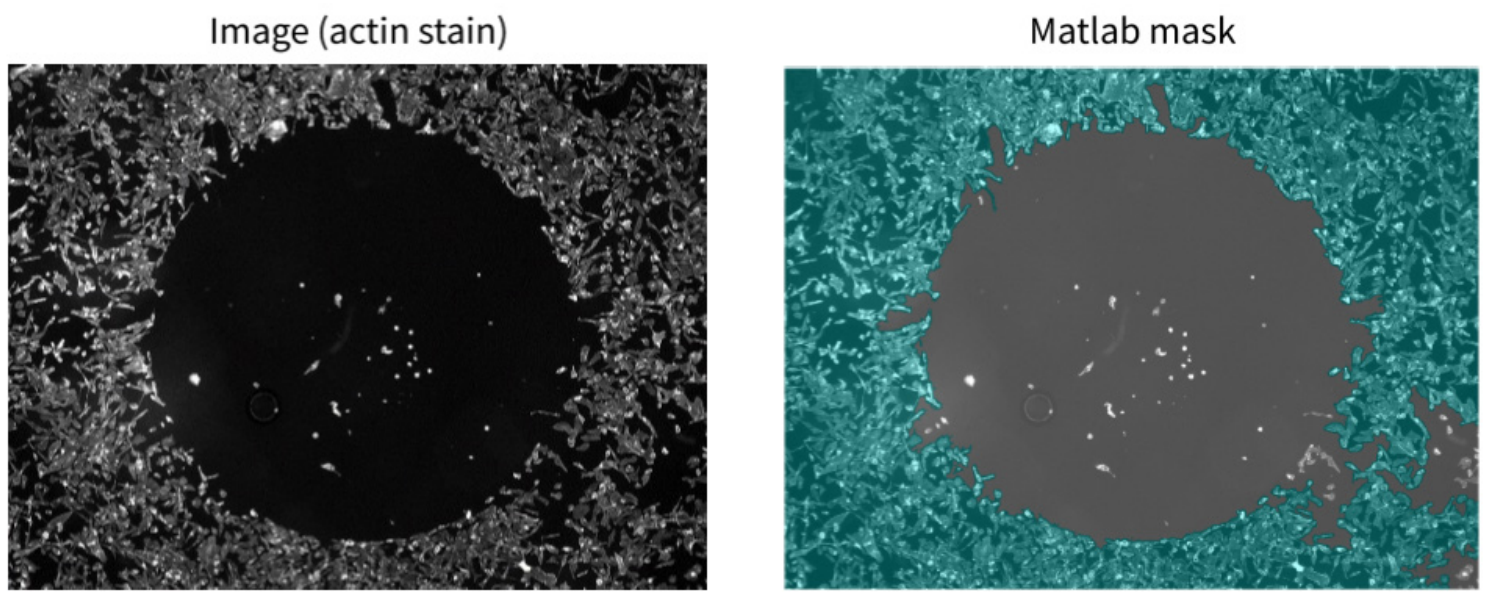

Figure 1. Sample images immediately after the stopper was removed. In order to identify the migration region, we took images of each well (left), then we select a mask that covers the area utilized by cells, highlighted in green (right).

where $c_{x}^{*}, c_{y}^{*}$, and $r^{*}$ are the optimal parameters of the migration region, $\boldsymbol{M}$ is the Matlab mask we are evaluating (i.e., a circle with center at coordinates $\left(c_{x}, c_{y}\right)$ and radius $\left.r\right)$, so $\sum_{m_{i, j} \in M} m_{i, j}$ is the sum of all the pixel intensities $\left(m_{i, j}\right)$ which belong to the mask $\boldsymbol{M}, \# \boldsymbol{M}$ is the cardinality of $\boldsymbol{M}$ (i.e., the number of pixels which belong to $\boldsymbol{M}$ ), and $p$ is a penalty parameter. If $p=1$, we have:

$$
\left[c_{x}^{*}, c_{y}^{*}, r^{*}\right]=\underset{M}{\operatorname{argmin}} \# \boldsymbol{M}-2 \sum_{m_{i, j} \in \boldsymbol{M}} m_{i, j}
$$

Hence, the maximization problem from Equation (1) is equivalent to the minimization represented in Equation (2) (when $p=1)$. From Equation (2), we can interpret the optimization performed by the genetic algorithm as finding "the largest circle which contains the least number of cell pixels." Figure 2 shows the optimal circular region selected by the genetic algorithm when the input is the image from Figure 1.

Percent of migration region covered by cells: We defined a metric to quantify the migration of MDA-MB-231 cells. This metric is $Q$, the percentage of migration pixels inside the migration region. We define a migration pixel as any pixel whose intensity value is greater than or equal to a threshold $T$. We chose $T$ equal to 1.25 times the median pixel intensity of the migration region immediately after the stopper was removed (i.e., the green region in Figure 2). This is:

$$
Q=\left(\sum_{i \in\left\{M^{*} \geq T\right\}} i\right) / \# \boldsymbol{M}^{*},
$$

Where $\boldsymbol{M}^{*}$ is the optimal circle defined by the three parameters $\left(\left[c_{x}^{*}, c_{y}^{*}, r^{*}\right]\right.$ from Equation (1) and Equation (2)) and the set $\left\{\boldsymbol{M}^{*} \geq T\right\}$ includes all of the pixels inside $\boldsymbol{M}^{*}$ with intensities higher than $T$.

\section{Preprint}

A previous version of this manuscript is available from bioRxiv: https://doi.org/10.1101/1305267

\section{Results and discussion}

To test the hypothesis that MDA-MB-231 cells' migration is reduced in the absence of AGR2, we designed an experiment (utilizing the cell migration assay described in the Methods section) with 5 experimental conditions: a positive control (untreated cells), a negative control (wells where the stopper was not removed), cells treated with $10 \mathrm{nM}$ of Taxol (a noncytotoxic dose level which prevents cell migration but does not promote cell death ${ }^{8}$ ), cells treated with a 1:50 dilution of AGR2-ab to inactivate eAGR2, and with a 1:50 dilution of IgG, a control antibody (Ctrl-Ab) which does not affect cell migration. Representative images from these conditions (i.e., replicate 1) are shown in Figure 3 (top).

For the untreated case and the control peptide we observe increased migration, with $46 \pm 2$ (mean \pm standard error of the mean) percent of the migration region covered in the untreated case and $42 \pm 1.7$ percent of the migration region covered in the control antibody case. We fail to reject the null hypothesis that these two are the sample means from the same distribution ( $\mathrm{p}$ value of 0.084 ). In the Taxol case, $21 \pm 1.9$ percent of the migration region is covered. We reject the null hypothesis that the mean of the Taxol population and the mean of the untreated case are sample means from the same distribution ( $p$ value of 8.16e-5). Similarly, for the AGR2-Ab case, $13 \pm 3.2$ percent of the migration region is covered. We reject the null hypothesis that the mean of the AGR2-Ab population and the mean of the untreated case are sample means from the same distribution ( $\mathrm{p}$ value of $2.19 \mathrm{e}-5$ ). Not only do we confirm the hypothesis that MDA-MB-231 cells' migration is reduced in the absence of AGR2, but our method allows for reproducible quantification of these qualitative observations. Furthermore, the algorithms used to compute $Q$ requires a single input from the user (a string with the names of the control experiments) and it can run on a desktop machine with Matlab (R2015a-2018a and above) installed.

\section{Conclusions and future work}

We have designed and implemented a pipeline for quantifying cell migration in vitro. It is worth noting that this metric 


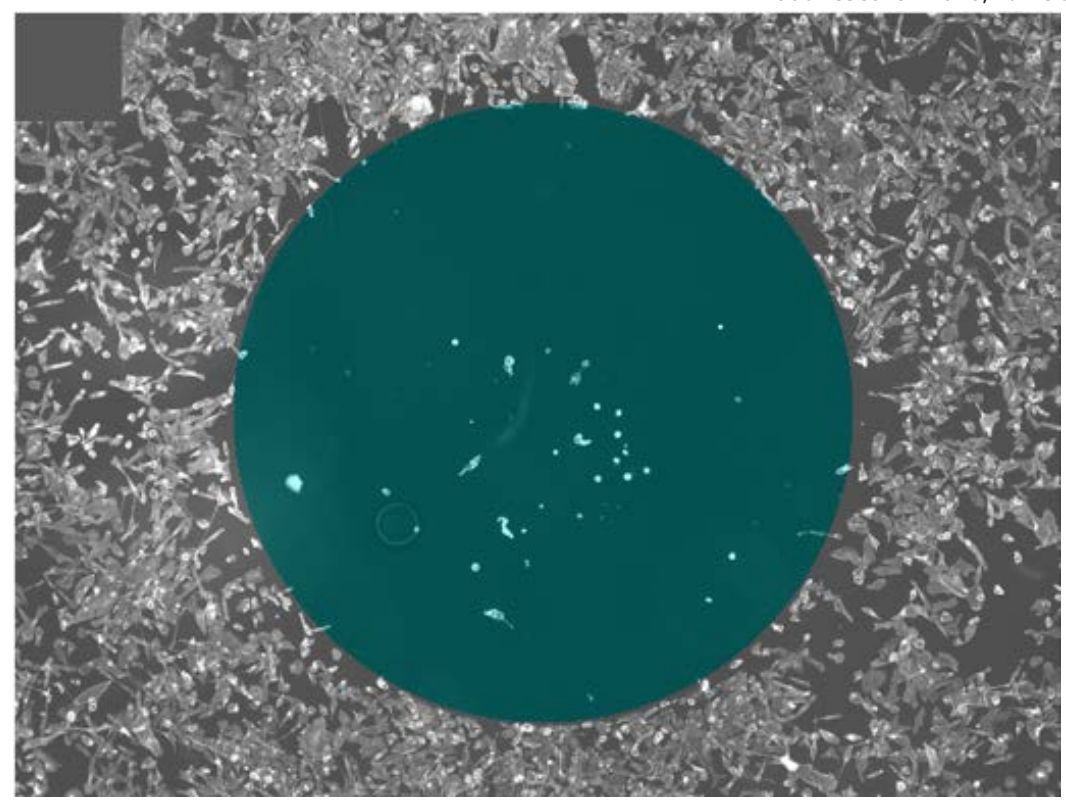

Figure 2. Automatically-selected migration region. The genetic algorithm selects the largest circle which contains the least number of masked pixels from the cell area mask. This optimal circle is the migration region.

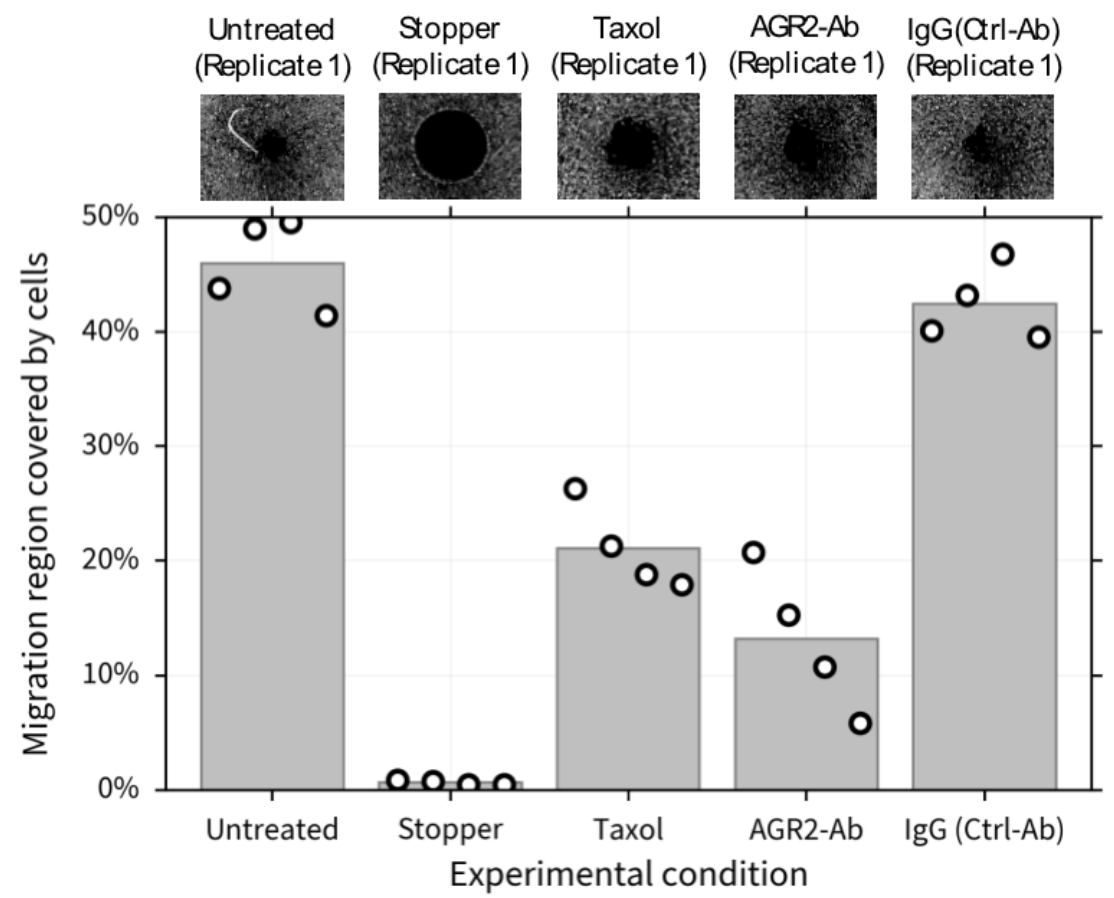

Figure 3. Quantifying cell migration. Representative images (replicate 1) of each condition are shown (top). 10nM of Taxol and the $10 \mu \mathrm{g} / \mathrm{mL}$ of the $\mathrm{H} 10$ peptide show similar levels of migration inhibition compared to the positive and negative controls. Our metric (bottom) allows us to quantify the qualitative results (top).

may not discern between cell motility and proliferation, hence in order to use it to estimate parameters for a mechanistic model, a parameter estimator such as CellPD ${ }^{9}$ should be used to estimate parameters of processes which decouple proliferation and motility ${ }^{10,11}$. However this metric is robust to image noise, open source, replicable, replicable and user friendly. In particular, we show that $\mathrm{H} 10$ aids in the reduction of migration of MDA-MB-231 cells by blocking sAGR2. This pipeline can be expanded to various cancer cell lines and model systems. 


\section{Data availability}

Raw, unedited microscope images from this analysis are available from the project GitHub repository: https://github.com/ edjuaro/cell-migration-quantification

\section{Software availability}

The source code used throughout this manuscript can be accessed in the public GitHub repository: https://github.com/ edjuaro/cell-migration-quantification.

Archived source code at time of publication can be found here: http://doi.org/10.5281/zenodo.1323923 ${ }^{12}$

This code is released under the permissive MIT license.

\section{Competing interests}

No competing interests were disclosed.

\section{Grant information}

This work was supported by the USC Center for Applied Molecular Medicine, the National Institutes of Health (Physical Sciences Oncology Center [5U54CA143907] for Multi-scale Complex Systems Transdisciplinary Analysis of Response to Therapy (MCSTART), and [1R01CA180149]), the Breast Cancer Research Foundation, the USC James H. Zumberge Research and Innovation Fund, and a USC Provost's PhD fellowship.

The funders had no role in study design, data collection and analysis, decision to publish, or preparation of the manuscript.
1. Jones $\mathrm{DH}$, Nakashima $\mathrm{T}$, Sanchez $\mathrm{OH}$, et al:: Regulation of cancer cell migration and bone metastasis by RANKL. Nature. 2006; 440(7084): 692-696. PubMed Abstract | Publisher Full Text

2. Brychtova V, Mohtar A, Vojtesek B, et al.: Mechanisms of anterior gradient-2 regulation and function in cancer. Semin Cancer Biol. 2015; 33: 16-24. PubMed Abstract | Publisher Full Text

3. Garri C, Howell S, Tiemann K, et al:: Identification, characterization and application of a new peptide against anterior gradient homolog 2 (AGR2) Oncotarget. 2018; 9(44): 27363-27379. PubMed Abstract | Publisher Full Text | Free Full Text

4. Kani K, Malihi PD, Jiang Y, et al:: Anterior gradient 2 (AGR2): blood-based biomarker elevated in metastatic prostate cancer associated with the neuroendocrine phenotype. Prostate. 2013; 73(3): 306-315. PubMed Abstract | Publisher Full Text

5. Fritzsche FR, Dahl E, Pahl S, et al:: Prognostic relevance of AGR2 expression in breast cancer. Clin Cancer Res. 2006; 12(6): 1728-1734. PubMed Abstract | Publisher Full Text

6. Price JT, Tiganis T, Agarwal A, et al.: Epidermal growth factor promotes MDA-MB231 breast cancer cell migration through a phosphatidylinositol $3^{\prime}$-kinase and phospholipase C-dependent mechanism. Cancer Res. 1999; 59(21): 5475-5478. PubMed Abstract
7. Juarez EF, Garri C, Ghaffarizadeh A, et al: Quantification of Cancer Cell Migration with an Integrated Experimental-Computational Pipeline. bioRxiv. Cold Spring Harbor Laboratory. 2017. Publisher Full Text

8. Zhang D, Yang R, Wang S, et al.: Paclitaxel: new uses for an old drug. Drug Des Devel Ther. 2014; 8: 279-84.

PubMed Abstract | Publisher Full Text | Free Full Text

9. Juarez EF, Lau R, Friedman SH, et al:: Quantifying differences in cell line population dynamics using CellPD. BMC Syst Biol. 2016; 10(1): 92. PubMed Abstract | Publisher Full Text | Free Full Text

10. Simpson MJ, Treloar KK, Binder BJ, et al.: Quantifying the roles of cell motility and cell proliferation in a circular barrier assay. J R Soc Interface. 2013; 10(82): 20130007.

PubMed Abstract | Publisher Full Text | Free Full Text

11. Treloar KK, Simpson MJ, McElwain DL, et al:: Are in vitro estimates of cell diffusivity and cell proliferation rate sensitive to assay geometry? J Theor Biol. 2014; 356: 71-84.

PubMed Abstract | Publisher Full Text

12. Juárez E: edjuaro/cell-migration-quantification: Pre-publication release (Version 1.0). Zenodo. 2018. http://www.doi.org/10.5281/zenodo.1323923 


\section{Open Peer Review}

\section{Current Peer Review Status: ? ?}

\section{Version 1}

Reviewer Report 18 September 2018

https://doi.org/10.5256/f1000research.17017.r38365

(c) 2018 Zaritsky A. This is an open access peer review report distributed under the terms of the Creative Commons Attribution License, which permits unrestricted use, distribution, and reproduction in any medium, provided the original work is properly cited.

\section{Assaf Zaritsky}

Department of Software and Information Systems Engineering, Ben-Gurion University of the Negev, Be'er Sheva, Israel

In their Software Tool Article "Quantification of cancer cell migration with an integrated experimental-computational pipeline", Juarez et al present a software to quantify circular monolayer migration assay. The assay itself is quite basic and widely used, although mostly not with circular geometry, which is used as an assumption by the segmentation algorithm. Multiple algorithms and tools exist for this task and the authors should compare their methods to alternatives. Quite a few details are missing making the algorithm very hard to read and interpret.

Specific major concerns / revisions:

The authors missed to cite a significant body of work methods for quantification of monolayer cell migration. Also, the experimental assay should be put into context - there are many alternatives assays to perform monolayer migration, Oris is just one example. As a "Software Tool" article, I am expecting the authors to benchmark using a ground truth annotated dataset and compare its performance to at least one of the existing alternatives. Here is one easy-to-use optional method, Gebäck et $\mathrm{al}^{1}$ (that does not use the information about the circular geometry).

The authors missed to discuss the limitations of their method. Specifically, the assumption that the pattern is circular does not fit most experimental settings.

Missing technical details:

The description of the algorithm is not clear to me. Is there a first stage of segmentation (thresholding?). Second stage of using the binary mask an input to optimize a fit to a circle as the initial geometry (is it the negative control?) using a genetic algorithm (GA). Third stage where segmentation is performed by simple thresholding and $\mathrm{Q}$ is calculated in relation to the initial circle as a measure to migration? This is very hard to interpret from the text. A major revision is essential. No details are provided for the GA. Many (most?) of the readers will not even know what is a GA. Moreover, there are practically no implementation details provided in the text.

I am not certain from the text whether $m_{-}$ij values are binary $(0 / 1)$ or the actual pixel 
intensity. In the latter case, the assumptions used to define the function to optimize with the GA do not necessarily hold as they are heavily dependent on the values and variability of the pixel's intensity in the background and foreground. The sentence "we can interpret the optimization performed by the genetic algorithm as finding "the largest circle which contains the least number of cell pixels." is not necessarily true when using the raw pixels intensities and has to at least be discussed - how was this equation derived? what were the assumptions? how to set the value of the parameter p? I would like to see the complete algorithm and how the equation was derived in any case.

What statistical tests were performed? Why do we see only 4 replication per experimental condition when the experiments were performed in a 96-well plate.

Other revisions and suggestions:

"A key aspect of metastasis is cell migration" 1 " this association of cell migration and metastasis is not established, definitely not through a 2D monolayer migration assay. Also, bone cancer (ref \#1) is very different than the model used for this study (breast cancer cell line). Monolayer cell migration assays are quite prevalent and a more relevant argument as motivation can be articulated. Overall, I suggest to tune-down the relevance to cancer throughout this article.

I would suggest to switch the order of the $2^{\text {nd }}$ and $3^{\text {rd }}$ paragraphs in the introduction. The focus of this article is the software tool. The experimental perturbation (AGR2) can come later (or better, not at all in the introduction).

In the same paragraph, why mention solely the AGR2 perturbation and ignore the Taxolperturbation. The authors should also mention somewhere that Taxol is a drug that inhibit microtubules.

"Our pipeline aids in the verification of well-established hypotheses" - it is the other way around: the known perturbation that is known to impair migration can be used to validate the method.

Typos / grammer:

"ZeoissObserver.Z1"

"First, the script first trains"

"If the user desires change the format of the images"

Missing detail:

"and applying a series of morphological operations"

"We then used a genetic algorithm to determine the coordinates of the center and the radius of a circle"

\section{References}

1. Gebäck T, Schulz MM, Koumoutsakos P, Detmar M: TScratch: a novel and simple software tool for automated analysis of monolayer wound healing assays.Biotechniques. 2009; 46 (4): 265-74 PubMed Abstract | Publisher Full Text

\section{Is the rationale for developing the new software tool clearly explained?} Partly

Is the description of the software tool technically sound? 
Partly

Are sufficient details of the code, methods and analysis (if applicable) provided to allow replication of the software development and its use by others?

Partly

Is sufficient information provided to allow interpretation of the expected output datasets and any results generated using the tool?

Yes

Are the conclusions about the tool and its performance adequately supported by the findings presented in the article?

Partly

Competing Interests: No competing interests were disclosed.

Reviewer Expertise: Computational cell dynamics Quantitative cell biology Cell migration High throughput phenotyping Computer vision application

I confirm that I have read this submission and believe that I have an appropriate level of expertise to confirm that it is of an acceptable scientific standard, however I have significant reservations, as outlined above.

Reviewer Report 29 August 2018

https://doi.org/10.5256/f1000research.17017.r37224

(C) 2018 El-Kebir M. This is an open access peer review report distributed under the terms of the Creative Commons Attribution License, which permits unrestricted use, distribution, and reproduction in any medium, provided the original work is properly cited.

\section{Mohammed El-Kebir}

Department of Computer Science, University of Illinois at Urbana-Champaign, Urbana, IL, USA

The authors introduce a tool for identifying the migration region in microscopy images from an in vitro experiment for metastasis in cell lines. Mathematically, the problem is to find the largest circle that contains the fewest cells. There are a couple of issues/inconsistencies in this paper that I will outline below.

1. Why do you need to train the tool on a negative control?

Please specify which parameters are being learned? Are you learning the value of parameter $p$ ? Please be specific.

2. More motivation is needed

Is the Oris cell migration assay the only assay where a circular stopper is used? How often is 
this assay used? Please describe this so that the reader can understand if your tool is widely applicable.

3. Description of related work is missing

In computational geometry, there is a problem that is called minimum enclosing circle (MEC). Please describe how and if your problem is different. (I'm not convinced it is - as there is a weighted version of MEC.)

4. Tool needs a name

Right now the tool is called code.m. Please give it a name.

\section{Notation}

Please don't use \#M to denote the cardinality of $\mathrm{M}$-- rather use $|\mathrm{M}|$.

Equation (1): don't use $c_{-} i, c_{-} j$ this clashes with $m_{-}\{i, j\}$. Use $c_{-} x$ and $c_{-} y$.

Equation (2): don't use $M$ in arg min. For consistency with (1) use c_x, c_y, r.

What is the relevance of $p=1$ ? Is this what you used in your experiments? If so, no need for this parameter in (1).

Is the rationale for developing the new software tool clearly explained?

Yes

Is the description of the software tool technically sound?

Partly

Are sufficient details of the code, methods and analysis (if applicable) provided to allow replication of the software development and its use by others?

No

Is sufficient information provided to allow interpretation of the expected output datasets and any results generated using the tool?

Yes

Are the conclusions about the tool and its performance adequately supported by the findings presented in the article?

Partly

Competing Interests: No competing interests were disclosed.

I confirm that I have read this submission and believe that I have an appropriate level of expertise to confirm that it is of an acceptable scientific standard, however I have significant reservations, as outlined above. 
The benefits of publishing with F1000Research:

- Your article is published within days, with no editorial bias

- You can publish traditional articles, null/negative results, case reports, data notes and more

- The peer review process is transparent and collaborative

- Your article is indexed in PubMed after passing peer review

- Dedicated customer support at every stage

For pre-submission enquiries, contact research@f1000.com 